\title{
Patterns and timing of sunlight exposure and risk of basal cell and squamous cell carcinomas of the skin - a case-control study
}

Michelle R lannacone ${ }^{1,8^{*}}$, Wei Wang ${ }^{2}$, Heather G Stockwell' , Kathleen O'Rourke ${ }^{2}$, Anna R Giuliano' Vernon K Sondak ${ }^{3}$, Jane L Messina ${ }^{3,4,5,6}$, Richard G Roetzheim7, Basil S Cherpelis ${ }^{5,6}$, Neil A Fenske ${ }^{4,5,6}$ and Dana E Rollison ${ }^{1}$

\begin{abstract}
Background: Non-melanoma skin cancer (NMSC), comprised of basal (BCC) and squamous (SCC) cell carcinomas, is the most common cancer in Caucasians. Ultraviolet radiation (UVR) exposure is the most important environmental risk factor for NMSC. However, the precise relationship between UVR and the risk of NMSC is complex, and the relationship may differ by skin cancer type.

Methods: A case-control study was conducted among Florida residents to investigate measures of patterns (intermittent vs. continuous) and timing (childhood vs. adulthood) of sunlight exposure in BCC and SCC. Participants included 218 BCC and 169 SCC cases recruited from a university dermatology clinic and 316 controls with no history of skin or other cancers.

Results: A history of blistering sunburn (a measure of intermittent sunlight exposure) was associated with both BCC $(\mathrm{OR}=1.96,95 \% \mathrm{Cl}=1.27-3.03)$ and SCC $(\mathrm{OR}=2.02,95 \% \mathrm{Cl}=1.22-3.33)$. Additionally, having a job in the sun for $\geq 3$ months for 10 years or longer (a measure of continuous sunlight exposure) was also associated with both BCC and SCC in our study population. With the exception of younger age at first blistering sunburn, measures of younger age at sunlight exposure tended to be associated with SCC, but not BCC risk.

Conclusions: Results from the current study suggest that sunlight exposure is associated with both BCC and SCC risk regardless of the pattern in which the exposure was received (i.e. intermittent vs. continuous). The data also suggest that sunlight exposure at a younger age may be more important for SCC but not BCC, however additional studies are needed to further characterize sunlight exposure-response relationships in different types of NMSC.
\end{abstract}

Keywords: Case-control, Sunlight, Patterns, Basal cell carcinoma, Squamous cell carcinoma, Non-melanoma skin cancer

\section{Background}

Non-melanoma skin cancer (NMSC), comprised of basal cell (BCC) and squamous cell (SCC) carcinomas, is the most common cancer in Caucasians, with more than one million new cases diagnosed annually in the United States (U.S.) alone [1]. While the mortality associated with NMSC is low $[2,3]$, patients with multiple NMSC's may

\footnotetext{
* Correspondence: iannaconem@fellows.iarc.fr

'Department of Cancer Epidemiology, Moffitt Cancer Center, Tampa, FL, USA ${ }^{8}$ Infections and Cancer Biology Group, International Agency for Research on Cancer, 150 cours Albert-Thomas, 69372 Lyon Cedex 08, France

Full list of author information is available at the end of the article
}

experience substantial morbidity, and treatment costs for NMSC are high at the national level [4]. Furthermore, a history of NMSC has been consistently associated with increased risk of subsequent primary cancers of other sites in studies from both the U.S. and Europe [5-13].

Ultraviolet radiation (UVR) exposure is considered the most important environmental risk factor for both $\mathrm{BCC}$ and SCC. However, the precise relationship between UVR and the risk of NMSC is complex, and the relationship may differ by skin cancer type. Starting in the late 1950s, researchers began to identify total (cumulative) outdoor sunlight exposure hours and sunlight exposure

\section{Biomed Central}


on working and non-working days [14-19] as risk factors for NMSC. Results from these studies suggested that $\mathrm{BCC}$ and SCC may have different exposure-response relationships with sunlight.

Patterns of sunlight exposure are continuous (i.e. persons working outdoors or living in a geographic region with a high annual UV index) or intermittent (i.e. persons working indoors and experiencing most of their sunlight exposure on the weekends or while vacationing to regions with a higher UV index than their place of residence). Timing of sunlight exposure refers to the period of life during which the majority of a person's sunlight exposure was experienced: childhood/adolescence, adulthood or both. Evidence from previous studies suggests that intermittent and childhood sunlight exposure may be important for the pathogenesis of BCC, whereas continuous, lifelong sunlight exposure may be important for SCC [20-24].

To further understand the relationship between sunlight exposure and risk of NMSC, a case-control study was conducted to investigate measures of patterns (intermittent vs. continuous) and timing (childhood vs. adulthood) of sunlight exposure in BCC and SCC. A major limitation of previously published studies is that they do not present direct comparisons between BCC and SCC from the same study population for associations with measures of patterns and timing of sunlight exposure. Therefore, differences in the observed associations may be explained by methodological inconsistencies in exposure measurement between study populations that investigate BCC or SCC alone. This is the first case-control study to simultaneously evaluate identical measures of patterns and timing of sunlight exposure as they are related to both BCC and SCC in the same U.S. population with high annual UVR exposure. The goal of the current study was to identify potential differences or similarities in sunlight exposure responses for BCC and SCC risk.

\section{Methods}

\section{Study design and population}

A clinic-based case-control study was conducted to evaluate the relationship between patterns and timing of sunlight exposure and risk of BCC and SCC. Complete study procedures have been described in detail elsewhere [25]. The University of South Florida (USF) Dermatology (D) clinic served as the primary location for recruitment of NMSC cases, comprised of patients with histologically-confirmed BCC or SCC. Control participants were recruited from the USF Family Medicine (FM) clinic and Moffitt's Lifetime Cancer Screening \& Prevention (LCS) clinic. Controls were individuals who self-reported no history of skin or other types of cancer and underwent a skin cancer screening exam at the time of study enrollment and screened negative for skin cancer. Additionally, patients that screened positive for a suspicious lesion, underwent a biopsy and were determined to be negative for skin cancer were also included as controls. All participants were recruited between October 30, 2006 and December 24, 2008. All participants provided written informed consent, and all study procedures were approved by the institutional review board at the University of South Florida.

Participation rates for the USF-D, the USF-FM, and LCS clinics were $80 \%, 47 \%$, and $65 \%$, respectively. There were no statistically significant differences in age or gender between those NMSC patients who agreed to participate and those that refused. A total of 358 controls, 245 BCC cases and 191 SCC cases were enrolled. The current study population was restricted to participants that provided complete questionnaire data (Controls: $\mathrm{n}=358$; BCC: $\mathrm{n}=222$; SCC: $\mathrm{n}=170$ ) and to White individuals and includes $218 \mathrm{BCC}$ and 169 SCC cases and 316 controls, between the ages of 18 and 80 .

\section{Exposure assessment}

Self-administered questionnaires were used to obtain information on sunlight exposures and potential confounding factors, including age, gender, ethnicity, education, eye and hair color, ever smoking, skin sensitivity to sunlight exposure (measured by skin reaction to one hour of sunlight exposure for the first time without sunscreen), and tanning ability (measured by change in skin color to repeated exposure to the summer sun). Patterns of sunlight exposure were measured using questions on history of blistering sunburn (yes/no), ever having a job in the sunlight for $\geq 3$ months (yes/no), the number of years with a job in the sunlight for $\geq 3$ months $(<1,1-5,6-10$, or $>10$ years $)$, lifetime frequency of tanning bed use ( $\leq 10,11-50,51-100,>100$ times), frequency of sunscreen application with a sunlight protection factor (SPF) of $\geq 15$ when outside for more than 15 minutes during the summer (always, often, sometimes, rarely, never), and the number of hours of mid-day sunlight exposure on a typical weekday $(<1,1-2$, $3-4,5-6$ hours) and weekend day $(<1,1-2,3-4,5-6$ hours) in the summer during one's teen years, twenties, thirties, and the past ten years prior to study enrollment. Experiencing blistering sunburn is considered a marker of intermittent sunlight exposure. Additionally, using sunscreen always/often or rarely/never is considered experiencing continuous sunlight exposure and using sunscreen some of time is considered intermittent sunlight exposure.

Timing of sunlight exposure was measured using questions on the age at which a blistering sunburn was experienced $(\leq 5,6-10,11-15,16-20,>20$ years), the number of moles larger than one quarter of an inch in 
diameter on the forearms (none, $<10,10-25,>25$ moles) and on the entire body (none, $<10,10-25,>25$ moles), the age at first tanning bed use $(\leq 15,16-20,>20$ years), and the number of hours of mid-day sunlight exposure on a typical weekday $(<1,1-2,3-4,5-6$ hours $)$ and weekend day $(<1,1-2,3-4,5-6$ hours $)$ in the summer during one's teen years, twenties, thirties, and in the past ten years prior to study enrollment. The presence of moles is considered an indicator of increased sunlight exposure in childhood or adolescence [26-31].

\section{Statistical analysis}

Demographic and skin cancer risk factors were compared between cases and controls using the chi-square test. To test whether measures of patterns or timing of sunlight exposure were associated with BCC or SCC, separate odds ratios (OR) and corresponding 95\% confidence intervals (CI) for each skin cancer type were calculated using unconditional logistic regression. Backward stepwise elimination was used to identify confounders from those factors previously shown to be associated with sunlight exposure and NMSC, including age (as a continuous variable), gender, ethnicity, education, eye, hair, and un-tanned skin color, cutaneous sensitivity and tanning ability to sunlight exposure, history of ever smoking, and alcohol consumption in the past year. Each factor retained in the model at $\mathrm{p}<.10$ was included in the final regression models; these factors included age, gender, ethnicity, education, eye and hair color, cutaneous sensitivity, tanning ability, and history of ever smoking. Variance inflation factors and Pearson correlation coefficients were estimated to identify multicollinear relationships between independent risk factors. No collinearity between co-factors and measures of patterns and timing of sunlight exposure was observed. Tests for trend in associations between measures of patterns and timing of sunlight exposure in $\mathrm{BCC} / \mathrm{SCC}$ were conducted by assigning ordinal values to each category and including the ordinal variable in the logistic regression model.

Factors associated with skin susceptibility to sunlight exposure have the potential to be factors on the causal pathway between UVR exposure and skin cancer. Therefore, to demonstrate the impact of these factors on the associations of interest, we present results from two different multivariate analyses. The first multivariate analysis adjusted for demographic and lifestyle factors only (i.e. age, gender, education, and history of ever smoking) and the second adjusted for demographic and lifestyle factors, as well as measures of skin susceptibility to sunlight exposure (i.e. ethnicity, eye and hair color, cutaneous sensitivity and tanning ability to sunlight exposure).

Utilizing data collected on the number of hours of sunlight exposure experienced on a typical weekday and weekend day during the summer in different time periods, summary scores were calculated. To measure cumulative sunlight exposure in early life (i.e. teens, twenties, and thirties), a median value was applied to each category of hours of sunlight exposure $(<1$ hour $=$ $0.5 ; 1-2$ hours $=1.5 ; 3-4$ hours $=3.5 ; 5-6$ hours $=5.5$ ) on a weekday and weekend day. The median values for weekday and weekend sunlight exposure were first summed for each age group, then summed across age groups (i.e. teens, twenties, and thirties) and finally divided into three categories: low, medium, and high. For intermittent sunlight exposure in early life, median values were once again applied to each category of hours of sunlight exposure. The ratio of median hours on a weekend day relative to that on a weekday was estimated separately for one's teen years, twenties, and thirties, summed across the three decades, and then divided into three groups: low (representing continuous sunlight exposure), medium, and high. Analyses including summary scores measuring sunlight exposure in early life were restricted to participants who were $\geq 40$ years of age.

For patterns of sunlight exposure by age at exposure (i.e. one's teens, twenties, thirties, and the 10 years prior to study enrollment), the participant was considered as having had continuous sunlight exposure if the reported number of hours of weekday sunlight exposure (1-2 or 3-6 hours) equaled that of weekend sunlight exposure (1-2 or 3-6 hours). However, if the reported number of hours of weekday sunlight exposure was less than that of weekend sunlight exposure, then the participant was considered as having intermittent sunlight exposure. Participants classified as having continuous or intermittent sunlight exposure were compared to participants with $<1$ hour of sunlight exposure on a typical weekday and weekend day. Daily sunlight exposure by age at exposure was measured by summing the median values of weekday and weekend hours of sunlight exposure and then dividing the values into three categories: low, medium, and high, independently for each time period.

The likelihood ratio test (LRT) was used to compare the statistical significance of the differences in effect sizes between BCC and SCC for each sun-related factor measured. Multiple testing was accounted for using Bonferroni correction. All analyses were performed using the SAS statistical software package (version 9.1.3; SAS Institute).

\section{Results}

Demographic, lifestyle, and skin susceptibility factors are presented for cases and controls in Table 1. Compared to controls, cases were significantly more likely to be older in age (BCC: $\mathrm{p}=<.0001$; SCC: $\mathrm{p}=<.0001$ ), male (BCC: $\mathrm{p}=<.0001$; SCC: $\mathrm{p}=<.0001$ ), less educated (BCC: $\mathrm{p}=0.0004 ;$ SCC: $\mathrm{p}=0.001)$, and ever smokers (BCC: 
Table 1 Demographic, lifestyle, and skin cancer risk factors in BCC and SCC cases and controls

\begin{tabular}{|c|c|c|c|c|c|}
\hline \multirow[b]{2}{*}{ Variable } & \multirow{2}{*}{$\begin{array}{l}\text { Controls } \\
(\mathrm{n}=316)\end{array}$} & \multicolumn{2}{|c|}{$\begin{array}{c}\text { BCC } \\
(n=218)\end{array}$} & \multicolumn{2}{|c|}{$\begin{array}{c}\text { SCC } \\
(n=169)\end{array}$} \\
\hline & & n (\%) & $p$-value ${ }^{1}$ & n (\%) & p-value \\
\hline $\begin{array}{l}\text { Age mean } \\
\text { (S.D.) }\end{array}$ & $55.6(11.8)$ & $62.8(11.9)$ & $<.0001$ & $64.8(9.6)$ & $<.0001$ \\
\hline \multicolumn{6}{|l|}{ Age (years) } \\
\hline $18-29$ & $9(2.9)$ & $1(0.5)$ & $<.0001$ & $1(0.6)$ & $<.0001$ \\
\hline $30-39$ & $21(6.7)$ & $6(2.8)$ & & $2(1.2)$ & \\
\hline $40-49$ & 55 (17.4) & $24(11.0)$ & & $10(5.9)$ & \\
\hline $50-59$ & $109(34.5)$ & $46(21.1)$ & & $30(17.8)$ & \\
\hline $60-69$ & $88(27.9)$ & $64(29.4)$ & & $68(40.2)$ & \\
\hline $70-80$ & $34(10.8)$ & 77 (35.3) & & $58(34.3)$ & \\
\hline \multicolumn{6}{|l|}{ Gender } \\
\hline Male & $117(37.0)$ & $133(61.0)$ & $<.0001$ & $108(63.9)$ & $<.0001$ \\
\hline Female & $199(63.0)$ & $85(39.0)$ & & $61(36.1)$ & \\
\hline \multicolumn{6}{|l|}{ Ethnicity } \\
\hline Non-Hispanic & $280(88.6)$ & $208(95.4)$ & 0.003 & $161(95.3)$ & 0.0003 \\
\hline Hispanic & $32(10.1)$ & $7(3.2)$ & & $2(1.2)$ & \\
\hline \multicolumn{6}{|l|}{ Education } \\
\hline$\leq 12$ years & $32(10.1)$ & $46(21.1)$ & 0.0004 & $36(21.3)$ & 0.001 \\
\hline$>12$ years & $280(88.6)$ & $168(77.1)$ & & $129(76.3)$ & \\
\hline \multicolumn{6}{|c|}{ Smoked 100 cigarettes } \\
\hline Never & $161(50.9)$ & $81(37.2)$ & 0.002 & $51(30.2)$ & $<.0001$ \\
\hline Ever & $154(48.7)$ & $134(61.5)$ & & $114(67.5)$ & \\
\hline \multicolumn{6}{|c|}{ Alcohol consumption } \\
\hline $\begin{array}{l}\geq 1 \text { drink in } \\
\text { past year }\end{array}$ & $274(86.7)$ & $177(81.2)$ & 0.09 & $130(76.9)$ & 0.02 \\
\hline $\begin{array}{l}\text { No drinks } \\
\text { in past year }\end{array}$ & $40(12.7)$ & $39(17.9)$ & & $35(20.7)$ & \\
\hline \multicolumn{6}{|l|}{ Eye color } \\
\hline Blue & $94(29.7)$ & $87(40.0)$ & 0.009 & $69(40.8)$ & 0.02 \\
\hline Green & $50(16.1)$ & $24(11.0)$ & & $25(14.8)$ & \\
\hline Hazel & $52(16.5)$ & $48(22.0)$ & & $31(18.3)$ & \\
\hline Light brown & $36(11.4)$ & $22(10.1)$ & & $18(10.7)$ & \\
\hline Dark brown & $80(25.3)$ & $35(16.1)$ & & $22(13.0)$ & \\
\hline \multicolumn{6}{|l|}{ Hair Color } \\
\hline Black/Brown & $245(77.5)$ & $152(69.7)$ & 0.04 & $113(66.9)$ & 0.02 \\
\hline Blonde/Red & $70(22.2)$ & $65(29.8)$ & & $53(31.4)$ & \\
\hline \multicolumn{6}{|c|}{ Color of un-tanned skin } \\
\hline White & $299(94.9)$ & $209(96.3)$ & 0.38 & $161(95.3)$ & 0.55 \\
\hline Brown & $15(4.8)$ & $7(3.2)$ & & $6(3.6)$ & \\
\hline \multicolumn{6}{|c|}{ Cutaneous sensitivity to sunlight exposure } \\
\hline $\begin{array}{l}\text { Sunburn } \\
\text { with blisters }\end{array}$ & $29(9.2)$ & $33(15.1)$ & 0.0001 & $22(13.0)$ & 0.005 \\
\hline $\begin{array}{l}\text { Sunburn } \\
\text { w/O blisters }\end{array}$ & $96(30.4)$ & $95(43.6)$ & & $71(42.0)$ & \\
\hline
\end{tabular}

Table 1 Demographic, lifestyle, and skin cancer risk factors in BCC and SCC cases and controls (Continued)

\begin{tabular}{lcccc}
\hline $\begin{array}{l}\text { Mild } \\
\text { sunburn/tan }\end{array}$ & $144(45.6)$ & $65(29.8)$ & $50(29.6)$ & \\
$\begin{array}{l}\text { Tan/no } \\
\text { change } \\
\text { color }\end{array}$ & $44(13.9)$ & $21(9.6)$ & $22(13.0)$ & \\
$\begin{array}{l}\text { Tanning ability to sunlight exposure } \\
\text { It is unable }\end{array} 22(7.0)$ & $15(6.9)$ & 0.04 & $26(15.4)$ & $<.0001$ \\
to tan & & & & \\
$\begin{array}{l}\text { Tan if you } \\
\text { work at it }\end{array}$ & $103(32.6)$ & $93(42.7)$ & & \\
It tans easily & $186(58.9)$ & $104(47.7)$ & & \\
\hline
\end{tabular}

${ }^{1} \mathrm{p}$-value for chi-square test.

$\mathrm{p}=0.002 ;$ SCC: $\mathrm{p}=<.0001)$. Additionally, NMSC cases were more likely to have light eye and hair color, a greater tendency to burn and a lesser tendency to tan from sunlight exposure, compared to controls.

Associations between patterns of sunlight exposure and NMSC are presented in Table 2. When adjusting for demographic and lifestyle factors only, a history of blistering sunburn was positively associated with both BCC $(\mathrm{OR}=1.96,95 \% \mathrm{CI}=1.27-3.03)$ and $\mathrm{SCC}(\mathrm{OR}=2.02,95 \%$ $\mathrm{CI}=1.22-3.33$ ). Ever having a job in the sunlight for $\geq 3$ months was significantly associated with SCC (OR = $1.73,95 \% \mathrm{CI}=1.06-2.83)$ but not $\mathrm{BCC}(\mathrm{OR}=1.38,95 \%$ $\mathrm{CI}=0.89-2.14)$. However, having a job in the sunlight for $\geq 3$ months for $>10$ years was significantly associated with both BCC $(\mathrm{OR}=2.14,95 \% \mathrm{CI}=1.12-4.11)$ and SCC $(\mathrm{OR}=2.54,95 \% \mathrm{CI}=1.23-5.28)$. With the exception of having a job in the sunlight for $>10$ years, the associations described above were no longer statistically significant after skin susceptibility co-factors were added to the multivariate models. When adjusting for demographic and lifestyle factors only, no associations were observed between levels of cumulative sunlight exposure or patterns of exposure in one's twenties or thirties and either BCC or SCC. However, after additional adjustment for measures of skin susceptibility, high levels of cumulative sunlight exposure were associated with BCC $(\mathrm{OR}=1.88,95 \% \mathrm{CI}=1.07-3.31)$ and medium $(\mathrm{OR}=2.36$, $95 \% \mathrm{CI}=1.22-4.57)$ and high $(\mathrm{OR}=2.47,95 \% \mathrm{CI}=1.25$ 4.91) levels of cumulative sunlight exposure were significantly associated with SCC, compared to low levels in early life. Additionally, sunlight exposure in one's twenties was associated with SCC regardless of the pattern of exposure; specifically, an OR of 2.99 (95\% CI $=1.19-7.48)$ was associated with continuous hours and an OR of 3.15 (95\% CI $=1.27-7.83)$ was associated with intermittent hours of exposure compared to $<1$ hour of sunlight exposure. Finally, in one's thirties, statistically significant associations were observed between intermittent hours of sunlight exposure and $\mathrm{BCC}(\mathrm{OR}=2.09,95 \% \mathrm{CI}=1.11$ - 
Table 2 Associations of measures of patterns of sunlight exposure in BCC and SCC cases and controls

\begin{tabular}{|c|c|c|c|c|c|c|c|}
\hline \multirow[b]{2}{*}{ Variable } & \multirow{2}{*}{$\frac{\text { Controls }(n=316)}{n(\%)}$} & \multicolumn{3}{|c|}{ Basal cell carcinoma $(n=218)$} & \multicolumn{3}{|c|}{ Squamous cell carcinoma $(n=169)$} \\
\hline & & n (\%) & OR $(95 \% \mathrm{Cl})^{1}$ & OR $(95 \% \mathrm{Cl})^{2}$ & $\mathrm{n}(\%)$ & OR $(95 \% \mathrm{Cl})^{1}$ & OR $(95 \% \mathrm{Cl})^{2}$ \\
\hline \multicolumn{8}{|l|}{ Blistering Sunburn } \\
\hline No & $101(32.3)$ & $54(25.0)$ & 1.00 (reference) & 1.00 (reference) & $38(23.0)$ & 1.00 (reference) & 1.00 (reference) \\
\hline Yes & $212(67.7)$ & $162(75.0)$ & $1.96(1.27-3.03)$ & $1.56(0.96-2.54)$ & $127(77.0)$ & $2.02(1.22-3.33)$ & $1.24(0.71-2.18)$ \\
\hline \multicolumn{8}{|l|}{ Job in sun $\geq 3$ months } \\
\hline No & $227(72.8)$ & $120(55.3)$ & 1.00 (reference) & 1.00 (reference) & $86(51.8)$ & 1.00 (reference) & 1.00 (reference) \\
\hline Yes & $85(27.2)$ & $97(44.7)$ & $1.38(0.89-2.14)$ & $1.31(0.81-2.12)$ & $80(48.2)$ & $1.73(1.06-2.83)$ & $1.72(0.99-2.97)$ \\
\hline \multicolumn{8}{|l|}{ \# years with job } \\
\hline$\leq 10$ & $57(18.7)$ & $47(22.3)$ & $1.17(0.70-1.94)$ & $1.07(0.61-1.86)$ & $44(27.0)$ & $1.64(0.94-2.86)$ & $1.64(0.88-3.07)$ \\
\hline \multirow[t]{2}{*}{$>10$} & $21(6.9)$ & $44(20.9)$ & $2.14(1.12-4.11)$ & $2.12(1.05-4.27)$ & $33(20.2)$ & $2.54(1.23-5.28)$ & $2.36(1.07-5.20)$ \\
\hline & & & $p_{\text {trend }}=0.03$ & $p_{\text {trend }}=0.06$ & & $p_{\text {trend }}=0.01$ & $p_{\text {trend }}=0.02$ \\
\hline Lifetime tanning bed use & $209(71.3)$ & $175(82.5)$ & 1.00 (reference) & 1.00 (reference) & $127(80.9)$ & 1.00 (reference) & 1.00 (reference) \\
\hline Never used & $44(15.0)$ & $25(11.8)$ & $0.99(0.56-1.76)$ & $0.99(0.53-1.82)$ & $18(11.5)$ & $1.01(0.52-1.98)$ & $0.80(0.38-1.71)$ \\
\hline $1-10$ times & $40(13.7)$ & $12(5.7)$ & $0.64(0.30-1.36)$ & $0.64(0.30-1.36)$ & $12(7.6)$ & $1.67(0.75-3.73)$ & $1.85(0.74-4.62)$ \\
\hline$>10$ times & & & $\mathrm{p}_{\text {trend }}=0.32$ & $p_{\text {trend }}=0.20$ & & $p_{\text {trend }}=0.29$ & $p_{\text {trend }}=0.40$ \\
\hline \multicolumn{8}{|l|}{ Apply $\mathrm{SPF}^{3} \geq 15$} \\
\hline Always/often & $124(39.6)$ & $82(37.8)$ & 1.00 (reference) & 1.00 (reference) & $53(32.5)$ & 1.00 (reference) & 1.00 (reference) \\
\hline Sometimes & $98(31.3)$ & $59(27.2)$ & $0.79(0.50-1.26)$ & $0.87(0.52-1.45)$ & $56(34.4)$ & $0.83(0.49-1.42)$ & $0.86(0.47-1.59)$ \\
\hline Rarely/never & $91(29.1)$ & $76(35.0)$ & $0.83(0.52-1.32)$ & $0.93(0.56-1.54)$ & $54(33.1)$ & $0.79(0.46-1.36)$ & $0.87(0.48-1.60)$ \\
\hline \multicolumn{8}{|c|}{ Cumulative sunlight exposure } \\
\hline Low & $87(32.3)$ & $52(27.4)$ & 1.00 (reference) & 1.00 (reference) & $35(22.7)$ & 1.00 (reference) & 1.00 (reference) \\
\hline Medium & $92(34.2)$ & $54(28.4)$ & $1.02(0.61-1.69)$ & $1.42(0.81-2.50)$ & $57(37.0)$ & $1.49(0.84-2.64)$ & $2.36(1.22-4.57)$ \\
\hline High & $90(33.5)$ & $84(44.2)$ & $1.37(0.83-2.27)$ & $1.88(1.07-3.31)$ & $62(40.3)$ & $1.59(0.88-2.87)$ & $2.47(1.25-4.91)$ \\
\hline \multicolumn{8}{|c|}{ Intermittent sunlight exposure } \\
\hline Low & $91(33.8)$ & $80(42.1)$ & 1.00 (reference) & 1.00 (reference) & $59(38.3)$ & 1.00 (reference) & 1.00 (reference) \\
\hline Medium & $84(31.2)$ & $52(27.4)$ & $0.99(0.60-1.64)$ & $1.26(0.72-2.22)$ & $46(29.9)$ & $1.25(0.71-2.20)$ & $1.58(0.83-3.00)$ \\
\hline High & $94(34.9)$ & $58(30.5)$ & $1.15(0.70-1.88)$ & $1.23(0.72-2.10)$ & $49(31.8)$ & $1.48(0.85-2.58)$ & $1.57(0.83-2.94)$ \\
\hline \multicolumn{8}{|l|}{ Patterns by age at exposure } \\
\hline \multicolumn{8}{|l|}{ Teens } \\
\hline$<1$ hour & $18(6.0)$ & $12(6.0)$ & 1.00 (reference) & 1.00 (reference) & $4(2.5)$ & 1.00 (reference) & 1.00 (reference) \\
\hline Continuous hours & $151(50.0)$ & $113(56.8)$ & $1.04(0.45-2.41)$ & $0.97(0.38-2.48)$ & $96(60.4)$ & $2.33(0.69-7.90)$ & $1.76(0.48-6.47)$ \\
\hline Intermittent hours & $133(44.0)$ & $74(37.2)$ & $1.08(0.46-2.54)$ & $1.10(0.42-2.83)$ & $59(37.1)$ & $2.26(0.66-7.78)$ & $1.86(0.50-6.94)$ \\
\hline
\end{tabular}


Table 2 Associations of measures of patterns of sunlight exposure in BCC and SCC cases and controls (Continued)

\begin{tabular}{|c|c|c|c|c|c|c|c|}
\hline$<1$ hour & $34(11.3)$ & $18(9.0)$ & 1.00 (reference) & 1.00 (reference) & $11(6.9)$ & 1.00 (reference) & 1.00 (reference) \\
\hline Continuous hours & $102(33.9)$ & $91(45.5)$ & $1.36(0.68-2.71)$ & $1.58(0.75-3.36)$ & $74(46.5)$ & $2.01(0.86-4.67)$ & $2.99(1.19-7.48)$ \\
\hline Intermittent hours & $165(54.8)$ & $91(45.5)$ & $1.30(0.66-2.56)$ & $1.56(0.74-3.26)$ & $74(46.5)$ & $2.11(0.92-4.88)$ & $3.15(1.27-7.83)$ \\
\hline \multicolumn{8}{|l|}{ Thirties } \\
\hline$<1$ hour & $60(20.5)$ & 27 (13.6) & 1.00 (reference) & 1.00 (reference) & 18 (11.3) & 1.00 (reference) & 1.00 (reference) \\
\hline Continuous hours & $85(29.0)$ & 79 (39.9) & $1.31(0.72-2.40)$ & $1.77(0.90-3.49)$ & $69(43.4)$ & $1.55(0.77-3.10)$ & $2.25(1.02-4.94)$ \\
\hline Intermittent hours & $148(50.5)$ & $92(46.5)$ & $1.38(0.79-2.41)$ & $2.09(1.11-3.93)$ & $72(45.3)$ & $1.47(0.76-2.85)$ & $1.95(0.92-4.12)$ \\
\hline \multicolumn{8}{|l|}{ Past 10 years } \\
\hline$<1$ hour & $63(28.6)$ & $52(30.2)$ & 1.00 (reference) & 1.00 (reference) & 49 (33.1) & 1.00 (reference) & 1.00 (reference) \\
\hline Continuous hours & 74 (33.6) & $83(48.3)$ & $0.88(0.51-1.52)$ & $1.14(0.62-2.12)$ & $64(43.2)$ & $0.81(0.46-1.42)$ & $1.35(0.69-2.64)$ \\
\hline Intermittent hours & $83(37.7)$ & 37 (21.5) & $0.57(0.32-1.03)$ & $0.67(0.35-1.28)$ & 35 (23.6) & $0.60(0.33-1.10)$ & $0.93(0.46-1.89)$ \\
\hline
\end{tabular}

${ }^{1}$ Odds ratios (OR) and $95 \%$ confidence intervals (Cl) adjusted for age, gender, education, and history of ever smoking.

${ }^{2} \mathrm{OR}$ and $95 \% \mathrm{Cl}$ adjusted for age, gender, education, history of ever smoking, ethnicity, eye and hair color, cutaneous sensitivity and tanning ability to sunlight exposure.

$\mathrm{SPF}=$ sun protection factor. 
Table 3 Associations of measures of timing of sunlight exposure in BCC and SCC cases and controls

\begin{tabular}{|c|c|c|c|c|c|c|c|}
\hline \multirow[b]{2}{*}{ Variable } & \multirow{2}{*}{$\frac{\text { Controls }(n=316)}{n(\%)}$} & \multicolumn{3}{|c|}{ Basal cell carcinoma $(n=218)$} & \multicolumn{3}{|c|}{ Squamous cell carcinoma $(n=169)$} \\
\hline & & n (\%) & OR $(95 \% \mathrm{Cl})^{1}$ & OR $(95 \% \mathrm{Cl})^{2}$ & $\mathrm{n}(\%)$ & OR $(95 \% \mathrm{Cl})^{1}$ & OR $(95 \% \mathrm{CI})^{2}$ \\
\hline \multicolumn{8}{|c|}{ \# of moles on forearms } \\
\hline None & $220(71.4)$ & $155(71.8)$ & 1.00 (reference) & 1.00 (reference) & $115(69.7)$ & 1.00 (reference) & 1.00 (reference) \\
\hline$<10$ & $80(26.0)$ & $53(24.5)$ & $0.84(0.54-1.31)$ & $0.65(0.40-1.06)$ & $39(23.6)$ & $0.92(0.56-1.52)$ & $0.94(0.54-1.64)$ \\
\hline \multirow[t]{2}{*}{$\geq 10$} & $8(2.6)$ & $8(3.7)$ & $1.65(0.57-4.77)$ & $1.75(0.55-5.61)$ & $11(6.7)$ & $3.27(1.12-9.58)$ & $2.69(0.75-9.59)$ \\
\hline & & & $\mathrm{p}_{\text {trend }}=0.96$ & $p_{\text {trend }}=0.45$ & & $\mathrm{p}_{\text {trend }}=0.25$ & $p_{\text {trend }}=0.43$ \\
\hline \multicolumn{8}{|c|}{ \# of moles on entire body } \\
\hline None & $118(39.1)$ & $79(36.9)$ & 1.00 (reference) & 1.00 (reference) & $56(34.1)$ & 1.00 (reference) & 1.00 (reference) \\
\hline$<10$ & $147(48.7)$ & $107(50.0)$ & $1.11(0.73-1.67)$ & $1.03(0.66-1.60)$ & $76(46.3)$ & $1.15(0.72-1.86)$ & $1.22(0.71-2.09)$ \\
\hline \multirow[t]{2}{*}{$\geq 10$} & $37(12.3)$ & $28(13.1)$ & $1.18(0.64-2.19)$ & $1.06(0.55-2.04)$ & $32(19.5)$ & $2.12(1.11-4.06)$ & $2.16(1.03-4.52)$ \\
\hline & & & $p_{\text {trend }}=0.54$ & $p_{\text {trend }}=0.86$ & & $p_{\text {trend }}=0.04$ & $p_{\text {trend }}=0.06$ \\
\hline \multicolumn{8}{|c|}{ Age at $1^{\text {st }}$ blistering sunburn } \\
\hline None & $101(32.9)$ & $54(25.2)$ & 1.00 (reference) & 1.00 (reference) & $38(23.5)$ & 1.00 (reference) & 1.00 (reference) \\
\hline$<10$ years & $62(20.2)$ & $47(22.0)$ & $1.97(1.14-3.42)$ & $1.35(0.73-2.49)$ & $46(28.4)$ & $2.25(1.22-4.13)$ & $1.07(0.53-2.15)$ \\
\hline $10-20$ years & $108(35.2)$ & $84(39.3)$ & $2.15(1.32-3.52)$ & $1.73(1.00-2.99)$ & $65(40.1)$ & $2.37(1.34-4.21)$ & $1.62(0.86-3.04)$ \\
\hline \multirow[t]{2}{*}{$>20$ years } & $36(11.7)$ & $29(13.6)$ & $1.71(0.89-3.28)$ & $1.67(0.83-3.37)$ & $13(8.0)$ & $0.96(0.42-2.20)$ & $0.81(0.33-2.01)$ \\
\hline & & & $\mathrm{p}_{\text {trend }}=0.01$ & $p_{\text {trend }}=0.05$ & & $p_{\text {trend }}=0.17$ & $p_{\text {trend }}=0.53$ \\
\hline \multicolumn{8}{|c|}{ Age at $1^{\text {st }}$ tanning bed use } \\
\hline Never used & $209(67.0)$ & $175(80.3)$ & 1.00 (reference) & 1.00 (reference) & $127(76.5)$ & 1.00 (reference) & 1.00 (reference) \\
\hline$\leq 20$ years & $38(12.2)$ & $20(9.2)$ & $1.09(0.58-2.06)$ & $1.10(0.55-2.18)$ & $23(13.9)$ & $1.97(1.01-3.85)$ & $1.97(0.91-4.27)$ \\
\hline \multirow[t]{2}{*}{$>20$ years } & 65 (20.8) & $23(10.6)$ & $0.64(0.64-1.12)$ & $0.56(0.30-1.05)$ & $16(9.6)$ & $0.77(0.40-1.50)$ & $0.78(0.37-1.65)$ \\
\hline & & & $p_{\text {trend }}=0.46$ & $p_{\text {trend }}=0.33$ & & $p_{\text {trend }}=0.96$ & $p_{\text {trend }}=0.98$ \\
\hline
\end{tabular}

Daily sunlight exposure by age at exposure

Teens

\begin{tabular}{|c|c|c|c|c|c|c|c|}
\hline Low & $63(20.9)$ & 27 (13.6) & 1.00 (reference) & 1.00 (reference) & $21(13.2)$ & 1.00 (reference) & 1.00 (reference) \\
\hline Medium & $104(34.4)$ & $68(34.2)$ & $1.28(0.71-2.30)$ & $1.18(0.62-2.25)$ & 55 (34.6) & $1.24(0.64-2.42)$ & $0.94(0.45-1.97)$ \\
\hline High & $135(44.7)$ & $104(52.3)$ & $1.38(0.78-2.43)$ & $1.47(0.78-2.77)$ & $83(52.2)$ & $1.43(0.75-2.73)$ & $1.40(0.68-2.89)$ \\
\hline \multicolumn{8}{|l|}{ Twenties } \\
\hline Low & $121(40.2)$ & $62(31.0)$ & 1.00 (reference) & 1.00 (reference) & $53(33.3)$ & 1.00 (reference) & 1.00 (reference) \\
\hline Medium & $114(37.9)$ & 79 (39.5) & $1.20(0.77-1.88)$ & $1.35(0.82-2.21)$ & $51(32.1)$ & $0.82(0.49-1.37)$ & $0.97(0.54-1.73)$ \\
\hline High & $66(21.9)$ & $59(29.5)$ & $1.22(0.73-2.31)$ & $1.31(0.75-2.31)$ & 55 (34.6) & $1.40(0.80-2.44)$ & $1.56(0.83-2.91)$ \\
\hline \multicolumn{8}{|l|}{ Thirties } \\
\hline Low & $152(51.9)$ & $87(43.9)$ & 1.00 (reference) & 1.00 (reference) & $64(40.3)$ & 1.00 (reference) & 1.00 (reference) \\
\hline Medium & $103(35.2)$ & $63(31.8)$ & $0.90(0.58-1.39)$ & $0.98(0.60-1.58)$ & $62(39.0)$ & $1.08(0.66-1.75)$ & $1.36(0.78-2.37)$ \\
\hline High & $38(13.0)$ & $48(24.2)$ & $1.20(0.68-2.10)$ & $1.28(0.69-2.36)$ & $33(20.8)$ & $1.15(0.61-2.18)$ & $1.30(0.63-2.68)$ \\
\hline \multicolumn{8}{|c|}{ Past 10 years } \\
\hline Low & $126(57.3)$ & $76(44.2)$ & 1.00 (reference) & 1.00 (reference) & $80(54.1)$ & 1.00 (reference) & 1.00 (reference) \\
\hline Medium & 65 (29.5) & $59(34.3)$ & $1.05(0.64-1.74)$ & $1.15(0.66-2.01)$ & $40(27.0)$ & $0.77(0.45-1.31)$ & $1.13(0.61-2.10)$ \\
\hline High & $29(13.2)$ & $37(21.5)$ & $1.41(0.74-2.68)$ & $1.62(0.79-3.30)$ & $28(18.9)$ & $1.16(0.59-2.25)$ & $1.57(0.73-3.36)$ \\
\hline
\end{tabular}

${ }^{1}$ Odds ratios (OR) and 95\% confidence intervals $(\mathrm{Cl})$ adjusted for age, gender, education, and history of ever smoking.

${ }^{2} \mathrm{OR}$ and $95 \% \mathrm{Cl}$ adjusted for age, gender, education, history of ever smoking, cutaneous sensitivity and tanning ability to sunlight exposure.

3.93) while continuous hours of sunlight exposure were associated with SCC $(\mathrm{OR}=2.25,95 \% \mathrm{CI}=1.02-4.94)$, compared to $<1$ hour of exposure, when adjusting for skin susceptibility co-factors. Regardless of the covariates included in the multivariate models, no statistically significant associations in BCC or SCC were observed with tanning bed use, sunscreen use, levels of intermittent sunlight exposure in early life, and patterns of sunlight 
exposure in one's teens and the past ten years prior to study enrollment.

Table 3 presents the associations between measures of timing of sunlight exposure and BCC and SCC. When adjusting for demographic and lifestyle factors only, associations with SCC were observed for the presence of $\geq 10$ moles on the forearms $(\mathrm{OR}=3.27,95 \% \mathrm{CI}=1.12$ 9.58) and entire body ( $\mathrm{OR}=2.12,95 \% \mathrm{CI}=1.11-4.06)$, compared to no moles. Similar associations were not observed with BCC. Experiencing a blistering sunburn in young childhood or adolescence was significantly associated with both BCC $(<10$ years: $\mathrm{OR}=1.97,95 \%$ $\mathrm{CI}=1.14-3.42 ; 10-20$ years: $\mathrm{OR}=2.15,95 \% \mathrm{CI}=1.32$ $3.52)$ and $\mathrm{SCC}(<10$ years: $\mathrm{OR}=2.25,95 \% \mathrm{CI}=1.22-4.13$; 10-20 years: $\mathrm{OR}=2.37,95 \% \mathrm{CI}=1.34-4.21)$, compared to never experiencing blistering sunburn. SCC cases were more likely to begin using a tanning bed prior to age $20(\mathrm{OR}=1.97,95 \% \mathrm{CI}=1.01-3.85)$, compared to never users. No significant associations with BCC were observed for age at first tanning bed use. Elevated OR estimates were observed for high levels of daily sunlight exposure during the summer with $\mathrm{BCC}$ and SCC across all time periods, however, none of these associations achieved statistical significance (Table 3). When including measures of skin susceptibility to sunlight exposure to the multivariate models, little differences were observed in the magnitudes of associations between measures of timing of sunlight exposure and BCC/SCC.

\section{Discussion}

A clinic based case-control study was conducted to identify associations between patterns and timing of sunlight exposure and two types of NMSC, BCC and SCC. It has been suggested that $\mathrm{BCC}$ and $\mathrm{SCC}$ risk may differ by the patterns and timing in which sunlight exposure was received. Utilizing similar definitions of sunlight exposure (i.e. number of hours of sunlight exposure to define intermittent and continuous exposure) we investigated multiple measures of sunlight exposure in BCC and SCC simultaneously and did not observe differences in measures of intermittent and continuous patterns of sunlight exposure between the two types of skin cancer. For example, having a job in sun for $>10$ years and cumulative sunlight exposure in early life were associated with both BCC and SCC. In addition, the appearance of $\geq 10$ moles on the entire body was significantly associated with SCC but not BCC, although the LRT did not demonstrate statistically significant differences in the OR effect sizes in SCC versus BCC.

Findings from previous studies that aimed to quantify the association between the amount of sunlight exposure and NMSC suggested that intermittent sunlight exposure is associated with $\mathrm{BCC}[24,32]$ while chronic sunlight exposure is associated with SCC [17,20,33-36]. Two of three previous case-control studies [20,34,37] observed associations between SCC and history of blistering sunburn while no associations have been previously reported with BCC $[24,34,37,38]$. Blistering sunburn is believed to result from high doses of intense UVR exposure in short increments of time and is therefore considered a measure of intermittency. However, blistering sunburn is also a measure of cutaneous sensitivity to sunlight exposure and may explain the observed associations in our study population for both BCC and SCC when co-factors measuring skin susceptibility to sunlight exposure were excluded from the multivariate models.

It has been estimated that approximately $25 \%$ of lifetime sunlight exposure occurs before 18 years of age [39]. Young childhood and adolescence is considered a time period when individuals have greater vulnerability to toxic exposure, such as UVR [39]. Associations with first occurrence of blistering sunburn during childhood or adolescence (age periods prior to skin cancer diagnosis) were similar for BCC and SCC risk in our study population when adjusting for demographic and lifestyle factors but not skin susceptibility factors to sunlight exposure. Among residents of Western Australia, blistering sunburn between 10 to 14 years of age was associated with BCC [24] while sunburn between 35 to 39 years of age was associated with SCC [20]. Many epidemiologic studies have investigated the association between sunlight exposure in early childhood and nevus development and provide evidence that increasing sunlight exposure in early years of life is associated with melanocytic nevus development [26-31]. Since most nevi develop in childhood and early adolescence [26-31] and the number of moles on the body decrease with an increase in age [40-43] their presence in adulthood may be considered an indicator of high UV exposure in childhood. Self-reported presence of $\geq 10$ moles on the entire body were significantly and positively associated with SCC in our study population. Similar results were not observed for BCC. A limited number of studies have reported findings for the association between the presence of moles and NMSC, of which, one case-control study from Western Australia [44] and one prospective cohort study of U.S. male health professionals [45] observed a positive dose-response relationship between an increasing number of moles and BCC. In contrast, among adults from the U.S. [37], the presence of moles was not associated with either BCC or SCC risk.

The current study has some limitations. Clinic based study populations are not necessarily representative samples of the general population. Information on the sub-type of BCC diagnosis (superficial and nodular) was not available for the BCC cases included in the current study population. As reported by Pelucchi et al. [46], the risk of superficial versus nodular BCC may differ by 
patterns of sunlight exposure. If proven true, this may minimize the differences in the effect sizes observed between BCC and SCC cases in relation to measures of patterns of sunlight exposure. The ability to detect statistically significant associations was limited by the small sample size including participants with a wide age distribution, as well as by exposure variables with multiple strata and adjustment for multiple co-factors. Additionally, sunlight exposure was not assessed at the site of BCC or SCC diagnosis, as done in previous studies [20,24]. Depending on the site of skin cancer diagnosis, this may result in participants underestimating the amount of sunlight exposure to the site of skin cancer diagnosis which, in turn would attenuate the observed differences. The anatomical distributions of BCC and SCC on the face, ears/neck, arms/ hands, or other body parts are $76 \%$ and $39 \%, 12 \%$ and $14 \%, 3 \%$ and $21 \%$, and $9 \%$ and $27 \%$, respectively. Among BCC and SCC cases combined, $60 \%$ of skin cancers in our study population occurred on the face. Since the face is chronically exposed to sunlight exposure regardless of the outdoor activity or type of clothing being worn, this could result in cases under-reporting their sunlight exposure. We also did not collect information on sunlight exposure during holidays or recreational activities. It is difficult to compare results across studies for the relationship between sunlight exposure and skin cancer due mainly to inconsistencies and variations in the methods used to measure sunlight exposure.

The questionnaire utilized in the current study includes questions previously validated for use in a skin cancer study conducted in Arizona, US [37]. However, despite the validity, there are additional challenges in exposure ascertainment by use of self-administered questionnaire that should be noted, such as recall bias, misclassification of exposure, and bias due to missing data. Case-control studies are often subject to recall bias because cases tend to think about their exposures more carefully as they might relate to their current cancer diagnosis. In addition, self-reported measures of previous sunlight exposures may result in measurement error from difficulty in remembering habits in the past. However, there is no reason to believe that the type of NMSC (i.e. BCC vs. SCC) would influence patients to think differently about their past sunlight exposure that would affect the OR comparison between BCC and SCC cases. Additionally, no significant differences in age and gender were observed between BCC and SCC cases that completed the study questionnaire and those that did not and further investigations demonstrated no bias due to missing data in the effect measures between patterns and timing of sunlight exposure in NMSC. Unlike previous studies [20,24,36], we measured intermittency of sunlight exposure in the current study by assuming that weekend hours were "non-working" hours for our study population and we were unable to estimate "lifetime" sunlight exposure or consider the amount of ambient solar irradiance received by study participants. Additionally, with regards to occupational sunlight exposure, caution should be taken when interpreting the results. More specifically, having a job in the sun for $\geq 3$ months could involve indoor work up to 9 months of the year. However, as outlined in the introduction, intermittent sunlight exposure has been defined in previous studies as well as in the current study as sunlight exposure received mostly during non-working days (assuming non-working days is $\leq 2$ days per week) or during a traditional 2 day weekend or while vacationing to regions with a higher UV index than an individual's place of residence. Given this definition, a job in the sun for $\geq 3$ months is indicative of continuous sunlight exposure. However, it is also possible that having a job in the sun for $\geq 3$ months could involve initial intermittent sunlight exposure provided the individual's occupation included indoor work during the previous 9 months. However, for individuals reporting a job in the sun for $\geq 3$ months for $>10$ years it is possible to conclude that these individuals have had high levels of continuous sunlight exposure for a minimum of 10 years. Finally, caution should be taken when interpreting the observations between age at first tanning bed use and SCC. Younger age at first tanning bed use was associated with SCC, but not BCC, and older age at first use was not associated with either skin cancer type in our study. While this observation may suggest that sunlight exposure at an earlier age is more important for SCC than BCC risk, it may also be an indicator of higher cumulative lifetime UVR exposure and as previously discussed, it has been hypothesized that continuous, lifelong sunlight exposure increases the risk for SCC.

Strengths of the current study should also be noted; it is the first case-control study to formally evaluate measures of patterns and timing of sunlight exposure in NMSC in a high risk U.S. population as well as to present findings simultaneously for both BCC and SCC, allowing for direct comparisons of patterns and timing of sunlight by skin cancer type. The controls were screened for current signs of BCC and SCC by a nurse practitioner to avoid misclassification of case-control status that may result from self-reported data. This is an important strength of our study as a portion of the screened patients were included as cases.

Understanding how sunlight exposure responses may potentially differ by NMSC type is important for better educating the public in sun safe behaviors. Simply advising a reduction in sunlight exposure will not help reduce the incidence of NMSC if changes in sunlight exposure patterns are related to skin cancer development. For example, applying sunscreen while on vacation may decrease $\mathrm{BCC}$ risk associated with intermittent sunlight 
exposure, but may not impact the risk of SCC, which may be more strongly related with continuous sunlight exposure. Additional studies are needed to highlight similarities and differences in the exposure-response relationship of patterns and timing of sunlight exposure with BCC and SCC. Furthermore, standardized methods for measuring sunlight exposure should be established to enable comparisons across different study populations.

\section{Abbreviations}

NMSC: Non-melanoma skin cancer; BCC: Basal cell carcinoma;

SCC: Squamous cell carcinoma; UVR: Ultraviolet radiation; OR: Odds ratio;

Cl: Confidence interval; U.S.: United States; USF: University of South Florida;

D: Dermatology; FM: Family Medicine; LCS: Lifetime Cancer Screening; SPF: Sun protection factor.

\section{Competing interests}

The authors declare that they have no competing interests.

\section{Authors' contributions}

All authors of this research paper have directly participated in the planning, execution, or analysis of the study and have read an approved the final version submitted. Specifically, DER was the principle investigator that conceived the study, received grant funding and oversaw all study procedures, as well as contributed intellectually to the manuscript. MRI developed the concept of the manuscript, contributed significantly to the organization and writing of the manuscript, and contributed significantly to data collection and data analysis. WW, HGS, and KO contributed intellectually to the manuscript. ARG and VKS contributed to gaining grant funding and significantly contributed intellectually to the manuscript. JLM was the study pathologist and significantly contributed to the end point analysis. RGR, BSC, and NAF significantly contributed to data collection and intellectual review of the manuscript. All authors read and approved the final manuscript.

\section{Acknowledgements}

This case-control study was funded by a grant to DER from the state of Florida's James and Esther King Biomedical Research Program (06NIR-08). The authors are grateful to the supporting staff at the USF and LCS clinics for their assistance with patient recruitment, especially Kristen A. Jonathan, Jill Weber, and Carolyn Gerow.

\section{Author details}

'Department of Cancer Epidemiology, Moffitt Cancer Center, Tampa, FL, USA. ${ }^{2}$ Department of Epidemiology and Biostatistics, College of Public Health, University of South Florida, Tampa, FL, USA. ${ }^{3}$ Cutaneous Oncology Program, Moffitt Cancer Center, Tampa, FL, USA. ${ }^{4}$ Department of Pathology and Cell Biology, University of South Florida College of Medicine, Tampa, FL, USA. ${ }^{5}$ Department of Dermatology, University of South Florida College of Medicine, Tampa, FL, USA. 'Department of Cutaneous Surgery, University of South Florida College of Medicine, Tampa, FL, USA. ${ }^{7}$ Department of Family Medicine, University of South Florida College of Medicine, Tampa, FL, USA. ${ }^{8}$ Infections and Cancer Biology Group, International Agency for Research on Cancer, 150 cours Albert-Thomas, 69372 Lyon Cedex 08, France.

Received: 17 April 2012 Accepted: 17 July 2012

Published: 20 September 2012

\section{References}

1. Jemal A, Siegel R, Ward E, Hao Y, Xu J, Murray T, Thun MJ: Cancer statistics, 2008. CA Cancer J Clin 2008, 58(2):71-96.

2. Miller DL, Weinstock MA: Nonmelanoma skin cancer in the United States: incidence. J Am Acad Dermatol 1994, 30(5 Pt 1):774-778.

3. Weinstock MA: Epidemiologic investigation of nonmelanoma skin cancer mortality: the Rhode Island Follow-Back Study. J Invest Dermatol 1994, 102(6):6S-9S.

4. Housman $T S$, Feldman $S R$, Williford PM, Fleischer AB Jr, Goldman ND, Acostamadiedo JM, Chen GJ: Skin cancer is among the most costly of all cancers to treat for the Medicare population. J Am Acad Dermatol 2003, 48(3):425-429
5. Friedman GD, Tekawa IS: Association of basal cell skin cancers with other cancers (United States). Cancer Causes Control 2000, 11(10):891-897.

6. Frisch $M$, Hjalgrim $H$, Olsen $\mathrm{JH}$, Melbye M: Risk for subsequent cancer after diagnosis of basal-cell carcinoma. A population-based, epidemiologic study. Ann Intern Med 1996, 125(10):815-821.

7. Frisch $M$, Melbye $M$ : New primary cancers after squamous cell skin cancer. Am J Epidemiol 1995, 141(10):916-922.

8. Hemminki K, Jiang Y, Steineck G: Skin cancer and non-Hodgkin's lymphoma as second malignancies. markers of impaired immune function? Eur J Cancer 2003, 39(2):223-229.

9. Levi F, La Vecchia C, Te VC, Randimbison L, Erler G: Incidence of invasive cancers following basal cell skin cancer. Am J Epidemiol 1998, 147(8):722-726.

10. Levi F, Randimbison L, La Vecchia C, Erler G, Te VC: Incidence of invasive cancers following squamous cell skin cancer. Am J Epidemiol 1997 146(9):734-739.

11. Milan T, Pukkala E, Verkasalo PK, Kaprio J, Jansen CT, Koskenvuo M, Teppo L: Subsequent primary cancers after basal-cell carcinoma: A nationwide study in Finland from 1953 to 1995. Int J Cancer 2000, 87(2):283-288.

12. Rosenberg CA, Greenland P, Khandekar J, Loar A, Ascensao J, Lopez AM: Association of nonmelanoma skin cancer with second malignancy. Cancer 2004, 100(1):130-138.

13. Troyanova P, Danon S, Ivanova T: Nonmelanoma skin cancers and risk of subsequent malignancies: a cancer registry-based study in Bulgaria. Neoplasma 2002, 49(2):81-85.

14. Gafa L, Filippazzo MG, Tumino R, Dardanoni G, Lanzarone F, Dardanoni L: Risk factors of nonmelanoma skin cancer in Ragusa, Sicily: A casecontrol study. Cancer Causes Control 1991, 2(6):395-399.

15. Gellin GA, Kopf AW, Garfinkel L: Basal Cell Epithelioma. a Controlled Study of Associated Factors. Arch Dermatol 1965, 91:38-45.

16. Hunter DJ, Colditz GA, Stampfer MJ, Rosner B, Willett WC, Speizer FE: Risk factors for basal cell carcinoma in a prospective cohort of women. Ann Epidemiol 1990, 1(1):13-23.

17. Vitasa BC, Taylor HR, Strickland PT, Rosenthal FS, West S, Abbey H, Ng SK, Munoz B, Emmett EA: Association of nonmelanoma skin cancer and actinic keratosis with cumulative solar ultraviolet exposure in Maryland watermen. Cancer 1990, 65(12):2811-2817.

18. Ferreira FR, Nascimento $L F$, Rotta O: Risk factors for nonmelanoma skin cancer in Taubate, Sao Paulo, Brazil: a case-control study. Rev Assoc Med Bras 2011, 57(4):424-430.

19. Simic D, Situm M, Marijanovic I, Hadzigrahic N: Most common skin tumours in correlation with solar ultraviolet radiation in the area of West Herzegovina. Coll Antropol 2011, 35(4):1129-1134.

20. English DR, Armstrong BK, Kricker A, Winter MG, Heenan PJ, Randell PL Case-control study of sun exposure and squamous cell carcinoma of the skin. Int J Cancer 1998, 77(3):347-353.

21. Gallagher RP, Hill GB, Bajdik CD, Coldman AJ, Fincham S, McLean DI, Threlfall WJ: Sunlight exposure, pigmentation factors, and risk of nonmelanocytic skin cancer. II. Squamous cell carcinoma. Arch Dermatol 1995, 131(2):164-169.

22. Gallagher RP, Hill GB, Bajdik CD, Fincham S, Coldman AJ, McLean DI, Threlfall WJ: Sunlight exposure, pigmentary factors, and risk of nonmelanocytic skin cancer. I. Basal cell carcinoma. Arch Dermatol 1995, 131(2):157-163.

23. Gallagher RP, Spinelli JJ, Lee TK: Tanning beds, sunlamps, and risk of cutaneous malignant melanoma. Cancer Epidemiol Biomarkers Prev 2005, 14(3):562-566.

24. Kricker A, Armstrong BK, English DR, Heenan PJ: Does intermittent sun exposure cause basal cell carcinoma? a case-control study in Western Australia. Int J Cancer 1995, 60(4):489-494.

25. Rollison DE, lannacone MR, Messina JL, Glass LF, Giuliano AR, Roetzheim RG, Cherpelis BS, Fenske NA, Jonathan KA, Sondak VK: Case-control study of smoking and non-melanoma skin cancer. Cancer Causes Control 2012, 23(2):245-254.

26. Bauer J, Buttner P, Wiecker TS, Luther H, Garbe C: Risk factors of incident melanocytic nevi: a longitudinal study in a cohort of 1,232 young German children. Int I Cancer 2005, 115(1):121-126.

27. Darlington S, Siskind V, Green L, Green A: Longitudinal study of melanocytic nevi in adolescents. J Am Acad Dermatol 2002, 46(5):715-722.

28. Dulon $M$, Weichenthal $M$, Blettner $M$, Breitbart $M$, Hetzer $M$, Greinert $R$, Baumgardt-Elms C, Breitbart EW: Sun exposure and number of nevi in 5- to 6year-old European children. J Clin Epidemiol 2002, 55(11):1075-1081. 
29. Harrison SL, MacLennan R, Buettner PG: Sun exposure and the incidence of melanocytic nevi in young Australian children. Cancer Epidemiol Biomarkers Prev 2008, 17(9):2318-2324.

30. Oliveria SA, Satagopan JM, Geller AC, Dusza SW, Weinstock MA, Berwick M, Bishop M, Heneghan MK, Halpern AC: Study of Nevi in Children (SONIC): baseline findings and predictors of nevus count. Am J Epidemiol 2009, 169(1):41-53.

31. Pettijohn KJ, Asdigian NL, Aalborg J, Morelli JG, Mokrohisky ST, Dellavalle RP, Crane LA: Vacations to waterside locations result in nevus development in Colorado children. Cancer Epidemiol Biomarkers Prev 2009, 18(2):454-463.

32. Kricker A, Armstrong BK, English DR, Heenan PJ: A dose-response curve for sun exposure and basal cell carcinoma. Int J Cancer 1995, 60(4):482-488.

33. Aubry F, MacGibbon B: Risk factors of squamous cell carcinoma of the skin. A case-control study in the Montreal region. Cancer 1985, 55(4):907-911.

34. Han J, Colditz GA, Hunter DJ: Risk factors for skin cancers: a nested case-control study within the Nurses' Health Study. Int J Epidemiol 2006 35(6):1514-1521.

35. Perea-Milla Lopez E, Minarro-Del Moral RM, Martinez-Garcia C, Zanetti R, Rosso S, Serrano S, Aneiros JF, Jimenez-Puente A, Redondo M: Lifestyles, environmental and phenotypic factors associated with lip cancer: a case-control study in southern Spain. Br J Cancer 2003, 88(11):1702-1707.

36. Rosso S, Zanetti R, Martinez C, Tormo MJ, Schraub S, Sancho-Garnier H, Franceschi S, Gafa L, Perea E, Navarro C: The multicentre south European study 'Helios'. II: Different sun exposure patterns in the aetiology of basal cell and squamous cell carcinomas of the skin. Br J Cancer 1996, 73(11):1447-1454.

37. Foote JA, Harris RB, Giuliano AR, Roe DJ, Moon TE, Cartmel B, Alberts DS: Predictors for cutaneous basal- and squamous-cell carcinoma among actinically damaged adults. Int I Cancer 2001, 95(1):7-11.

38. Boyd AS, Shyr Y, King LE Jr: Basal cell carcinoma in young women: an evaluation of the association of tanning bed use and smoking. J Am Acad Dermatol 2002, 46(5):706-709.

39. Balk SJ: Ultraviolet Radiation: A Hazard to Children and Adolescents. Pediatrics 2011, 127(3):e791-e817.

40. Lasithiotakis KG, Kokolakis A, Giannikaki E, Krasagakis K, Tosca A: Factors associated with the prevalence of atypical nevus in a Mediterranean pigmented skin lesion clinic. Melanoma Res 2011, 21(5):469-473.

41. Mackie RM, English J, Aitchison TC, Fitzsimons CP, Wilson P: The number and distribution of benign pigmented moles (melanocytic naevi) in a healthy British population. Br J Dermatol 1985, 113(2):167-174.

42. Silva Idos S, Higgins CD, Abramsky T, Swanwick MA, Frazer J, Whitaker LM Blanshard ME, Bradshaw J, Apps JM, Bishop DT: Overseas sun exposure, nevus counts, and premature skin aging in young English women: a population-based survey. J Invest Dermatol 2009, 129(1):50-59.

43. Zalaudek I, Schmid K, Marghoob AA, Scope A, Manzo M, Moscarella E, Malvehy J, Puig S, Pellacani G, Thomas L, et al: Frequency of dermoscopic nevus subtypes by age and body site: a cross-sectional study. Arch Dermatol 2011, 147(6):663-670

44. Kricker A, Armstrong BK, English DR, Heenan PJ: Pigmentary and cutaneous risk factors for non-melanocytic skin cancer-a case-control study. Int J Cancer 1991, 48(5):650-662.

45. van Dam RM, Huang Z, Rimm EB, Weinstock MA, Spiegelman D, Colditz GA, Willett WC, Giovannucci E: Risk factors for basal cell carcinoma of the skin in men: results from the health professionals follow-up study. Am J Epidemiol 1999, 150(5):459-468

46. Pelucchi C, Di Landro A, Naldi L, La Vecchia C: Risk factors for histological types and anatomic sites of cutaneous basal-cell carcinoma: an italian case-control study. J Invest Dermatol 2007, 127(4):935-944.

doi:10.1186/1471-2407-12-417

Cite this article as: lannacone et al:: Patterns and timing of sunlight exposure and risk of basal cell and squamous cell carcinomas of the skin - a case-control study. BMC Cancer 2012 12:417.

\section{Submit your next manuscript to BioMed Central and take full advantage of:}

- Convenient online submission

- Thorough peer review

- No space constraints or color figure charges

- Immediate publication on acceptance

- Inclusion in PubMed, CAS, Scopus and Google Scholar

- Research which is freely available for redistribution

Submit your manuscript at www.biomedcentral.com/submit
C Biomed Central 